\title{
A qualitative approach to adsorption mechanism identification on microporous carbonaceous surfaces
}

\author{
Magda Ziółkowska ${ }^{1} \cdot$ Janina Milewska-Duda $^{1} \cdot$ Jan T. Duda $^{2}$
}

Received: 9 March 2015/Revised: 21 December 2015 / Accepted: 4 January 2016/Published online: 18 January 2016

(C) The Author(s) 2016. This article is published with open access at Springerlink.com

\begin{abstract}
The paper presents results of research on identification of localized and other adsorption mechanisms, on geometrically heterogeneous graphite-like carbonaceous surfaces. It attempts to get an insight into properties of individual adsorptive molecule movement near attractive adsorption sites, arising from adsorbent surface geometrical heterogeneities. In particular, a shape and volume of space occupied by the continuously moving molecule mass center are investigated. To this aim, kinematic equilibrium of the particle moving near a hypothetical microporous carbonaceous adsorbent wall is considered, and then compared with thermodynamic equilibrium. The proposed approach enables to examine effects of certain surface geometry on the shape and volume of space occupied by adsorbed particles, and so to outline temperature conditions for the localized adsorption mechanism predomination. Thus, it provides a cognitive basis to answer the question, what particular mechanism (localized or other-e.g. mobile) should be assumed for a class of adsorption systems in order to select the most appropriate mathematical adsorption model. Hence, it makes it possible for more reliable examination of real porous structures, based on adsorption measurements.
\end{abstract}

Electronic supplementary material The online version of this article (doi:10.1007/s10450-016-9756-2) contains supplementary material, which is available to authorized users.

Magda Ziółkowska

magda.ziolkowska@agh.edu.pl

1 Faculty of Energy and Fuels, AGH University of Science and Technology, Al. Mickiewicza 30, 30-059 Kraków, Poland

2 Faculty of Management, AGH University of Science and Technology, ul. Gramatyka 10, 30-251 Kraków, Poland
Keywords Adsorption - Adsorption mechanisms . Localized adsorption $\cdot$ Mobile adsorption

\section{Variables \\ $-$ \\ $\delta_{c}$ \\ $\varepsilon_{C-f k}$ \\ $\zeta_{A}$}

$\pi$

Fraction of one mole of individual particles of volatile phase, calculated as a remaining part $\left(1-w_{\text {max }}\right)$; dimensionless

$\sigma_{f f} \quad$ Adsorbate hard sphere core diameter $(\mathrm{nm})$

$\sigma_{C-f k} \quad$ Lennard-Jones potential intermolecular diameter for carbon atom interaction with adsorbate particle, at $k$-th position (nm)

$\tau_{i} \quad i$-th particle "temperature", average in phase space $v(\mathrm{~K})$

$\tau_{\text {ind }} \quad$ Temperature corresponding to a $V_{\text {ind }}(\mathrm{K})$

$\tau_{\max } \quad$ Temperature corresponding to a $V_{\max }(\mathrm{K})$

$a_{f i} \quad$ Specific surface area of a movement space of $i$-th adsorbate particle mass center $\left(\mathrm{nm}^{2}\right)$ Diameter of an equivalent sphere of volume $V_{f i}(\mathrm{~nm})$

Diameter of an equivalent sphere of volume $V_{p i}(\mathrm{~nm})$

$f\left(\tau_{i}, T\right) \quad$ Maxwell-Boltzmann distribution function at a given temperature $T$ of isothermal adsorption; dimensionless 
$J \quad$ Number of particles sharing occupied $\quad N_{\text {ind }}$ volume in a range $V_{\text {ind }}<V_{\text {mpi }}\left(\tau_{i}\right) \leq V_{\max }$; dimensionless

$k_{B}$

$k_{B} \tau(t)$

$k_{B} \tau_{i}$

$n_{\text {adm }}$

$n_{v}$

$p$

$p_{f i}$

$r_{C-f k}$

$s_{f i}$

$s_{p i}$

$t_{\text {int }}$

$u_{C-f k}$

$A, B, C, D$

$E_{a d h}$

$E_{\text {vap }}$

G

$\left(\frac{\mathrm{J}}{\mathrm{mol}}\right)$

$N_{A} \quad$ Avogadro constant $\left(\frac{1}{\mathrm{~mol}}\right)$

Averaged in time kinetic energy of two degrees of freedom $(\mathrm{K})$

Averaged in space $v$ kinetic energy of two degrees of freedom $(\mathrm{K})$

Admissible shots in Monte Carlo technique, respecting geometrical constraints of a modeled adsorbent; dimensionless

Successful shots in Monte Carlo technique, respecting geometrical constraints of a modeled adsorbent and additionally fulfilling a relation $\sum_{C} u_{C-f_{k}} \leq U_{b d r y}$;

dimensionless

Pressure, representing total effects of interactions of an adsorbate particle with volatile phase $(\mathrm{Pa})$

Effective pressure, representing total effects of interactions of an $i$-th individual adsorbate particle with volatile phase $(\mathrm{Pa})$ Lennard-Jones potential intermolecular distance for carbon atom interaction with adsorbate particle, at $k$-th position $(\mathrm{nm})$ Open surface limiting an $i$-th particle mass center movement in volume $V_{f i}\left(\mathrm{~nm}^{2}\right)$ Open surface limiting an $i$-th particle movement in volume $V_{p i}\left(\mathrm{~nm}^{2}\right)$ Time intervals of a molecule movement between collisions with other adsorptive molecules (s)

Time averages over a particle movement after a suitable lapse of time, frequently longer than $t_{\text {int }}(\mathrm{s})$

Lennard-Jones potential of carbon atom interaction with adsorbate particle, at $k$-th position $(\mathbf{J})$

Phase-space variables of an $i$-th particle mass center movement; dimensionless

\section{Riedel equation parameters; dimensionless} Effective molar adhesion energy for a pair of molecules being in a full contact $\left(\frac{\mathrm{J}}{\mathrm{mol}}\right)$ Cohesion (evaporation) energy of an adsorbate in its reference state $\left(\frac{\mathrm{J}}{\mathrm{mol}}\right)$ Free enthalpy of a thermodynamic system J $V_{f i}$
$Q_{A}$ $U_{m}\left(V_{m p i}\right)$

$U_{\text {madh }}\left(V_{\text {mpi }}\right)$

$V_{c}$

$V_{f i}$

Number of particles corresponding to a particular temperature $\tau_{i}$, fulfilling constraint $\tau_{i} \leq \tau_{\text {ind }}$; dimensionless Measurable monolayer adsorption energy $\left(\frac{\mathrm{J}}{\mathrm{mol}}\right)$

$R \quad$ Gas constant $\left(\frac{\mathrm{J}}{\mathrm{K} \cdot \mathrm{mol}}\right)$

$S_{a d s}$

$S_{\text {conf }}$

$S_{i}$

$T_{k}$

$T_{t}$

$T_{B}$

$T_{c}$

$U_{a d s}$

$U_{\text {avge }}(t)$

$U_{\text {avge }}\left(V_{f i}\right)$

$U_{\text {avge }}\left(V_{p i}\right)$

Entropy of thermodynamic system $\left(\frac{\mathrm{J}}{\mathrm{K} \cdot \mathrm{mol}}\right)$ Configurational entropy of thermodynamic system $\left(\frac{\mathrm{J}}{\mathrm{K} \cdot \mathrm{mol}}\right)$

$i$-th particle internal entropy in a thermodynamic system $\left(\frac{\mathrm{J}}{\mathrm{K} \cdot \mathrm{mol}}\right)$

Average temperature of adsorbed molecules at a kinematic equilibrium $(\mathrm{K})$

Average temperature of adsorbed molecules at a thermodynamic equilibrium $(\mathrm{K})$

Adsorbate boiling temperature $(\mathrm{K})$

Adsorbate critical temperature $(\mathrm{K})$

Total energy of adsorbate molecules $\left(\frac{\mathrm{J}}{\mathrm{mol}}\right)$

Averaged in time, solid-fluid potential $\left(\frac{\mathrm{J}}{\mathrm{mol}}\right)$

Averaged in space $v$, solid-fluid potential of the individual $i$-th particle mass center moving in volume $V_{f i}\left(\frac{\mathrm{J}}{\mathrm{mol}}\right)$

Averaged in space $v$, solid-fluid potential of an individual $i$-th particle moving in volume $V_{p i}\left(\frac{\mathrm{J}}{\mathrm{mol}}\right)$

$U_{b d r y}(t) \quad$ Averaged in time, boundary equipotential surface $\left(\frac{\mathrm{J}}{\mathrm{mol}}\right)$

$U_{b d r y}\left(V_{f i}\right) \quad$ Averaged in space $v$, boundary equipotential surface of an individual $i$-th particle mass center moving in volume $V_{f i}\left(\frac{\mathrm{J}}{\mathrm{mol}}\right)$

$U_{b d r y}\left(V_{p i}\right) \quad$ Averaged in space $v$, boundary equipotential surface of an individual $i$-th particle moving in volume $V_{p i}\left(\frac{\mathrm{J}}{\mathrm{mol}}\right)$

$U_{b d r y}\left(V_{p i}\right)$ related to one mole of particles with Eq. $9\left(\frac{\mathrm{J}}{\mathrm{mol}}\right)$

$U_{\text {avge }}\left(V_{p i}\right)$ related to one mole of particles with Eq. $9\left(\frac{\mathrm{J}}{\mathrm{mol}}\right)$

Adsorbate critical volume $\left(\frac{\mathrm{cm}^{3}}{\mathrm{~mol}}\right)$

Volume of an $i$-th individual particle mass center movement (free space) $\left(\mathrm{cm}^{3}\right)$ 


\begin{tabular}{|c|c|}
\hline$V_{\text {ind }}$ & $\begin{array}{l}\text { Maximum separated volume occupied by } \\
\text { one mole of individual particles }\left(\frac{\mathrm{cm}^{3}}{\mathrm{~mol}}\right)\end{array}$ \\
\hline$V_{\max }$ & $\begin{array}{l}\text { Maximum separated or shared with } \\
j \text { molecules volume, occupied by one mole } \\
\text { of individual particles }\left(\frac{\mathrm{cm}^{3}}{\mathrm{~mol}}\right)\end{array}$ \\
\hline$V_{p i}$ & $\begin{array}{l}\text { Volume of an } i \text {-th individual particle } \\
\text { movement }\left(\mathrm{cm}^{3}\right)\end{array}$ \\
\hline$V_{m f i}$ & $\begin{array}{l}\text { Volume } V_{f i} \text {, related to one mole of particles } \\
\left(\frac{\mathrm{cm}^{3}}{\mathrm{~mol}}\right)\end{array}$ \\
\hline$V_{m p i}$ & $\begin{array}{l}\text { Volume } V_{p i} \text {, related to one mole of particles } \\
\left(\frac{\mathrm{cm}^{3}}{\mathrm{~mol}}\right)\end{array}$ \\
\hline$V_{p}$ & $\begin{array}{l}\text { Total volume of adsorbate molecules in a } \\
\text { thermodynamic system }\left(\frac{\mathrm{cm}^{3}}{\mathrm{~mol}}\right)\end{array}$ \\
\hline$V_{m p}\left(T_{k}\right)$ & $\begin{array}{l}\text { Volume of adsorbate molecules at a } \\
\text { temperature of kinematic equilibrium } T_{k} \\
\left(\frac{\mathrm{cm}^{3}}{\mathrm{~mol}^{3}}\right)\end{array}$ \\
\hline$V_{m p}\left(T_{t}\right)$ & $\begin{array}{l}\text { Volume of adsorbate molecules at a } \\
\text { temperature of thermodynamic equilibrium } \\
T_{t}\left(\frac{\mathrm{cm}^{3}}{\mathrm{~mol}}\right)\end{array}$ \\
\hline$w_{\text {ind }}$ & $\begin{array}{l}\text { Localized mechanism fraction of a volatile } \\
\text { phase particles; dimensionless }\end{array}$ \\
\hline$w_{\max }$ & $\begin{array}{l}\text { Possible mobile mechanism (or other than } \\
\text { localized) fraction of a volatile phase } \\
\text { particles; dimensionless }\end{array}$ \\
\hline
\end{tabular}

\section{Introduction}

Practical identification of material porous structure from experimental adsorption measurements requires a mathematical description of adsorption mechanism. A number of theoretical investigations were elaborated in order to reliably describe the adsorption equilibrium, where different aspects of the adsorption mechanism are stressed (Dąbrowski 2001; Sing et al. 1985). Nevertheless, the mechanism itself is a basic assumption of any mathematical model, and its arbitrary selection is most commonly related to the surface description: heterogeneous for localized mechanism and homogeneous for others.

The classical approach is based on a lattice surface model that leads to the Langmuir (Langmuir 1916), Fowler-Guggenheim (Fowler and Guggenheim 1940) and BET formulas for localized adsorption, while the mobile adsorption is described by Volmer (Volmer 1925) or Hillde Boer (1968; Hill 1946) equations. On the other hand a potential theory of adsorption [for example DubininRadushkievitch model (Dubinin 1989)] is successfully employed, assuming formation of a quasi liquid like phase near the solid surface, i.e. based on mobile adsorption mechanism. Recently, this approach is intensively developed in the form of Density Functional Theory formalism [DFT, (Jagiełło and Olivier 2013; Lastoskie and Gubbins 2000)], where the adsorbate molecules are treated with the same formulae as describing the liquid bulk phase, but affected by the solid surface attractive forces.

Furthermore, in our earlier works we proposed a new approach for the localized adsorption modeling, in which a clustering based mechanism is exposed. From a formal viewpoint, it is a far generalization of BET multilayer adsorption (i.e. adsorbate clusters may be viewed as localized layers). Geometrical and energetic constraints of the clusters formation are taken into account, as well as configurational effects coming from the clusterization mechanism. Resultant general formula, referred to as a uniBET model may be adapted to random microporous structures, yielding LBET class adsorption models. The latter is applicable to the microporous heterogeneous structures examination, by employing a multivariant fitting procedure (Kwiatkowski et al. 2011, 2014).

An elaboration of a comprehensive mathematical model describing adsorption phenomena and taking into account localized and other mechanisms is almost virtually impossible [due to chemical and structural diversity of the porous materials (Jagiełło and Olivier 2013)]. Hence, the mechanism in the abovementioned models is commonly assumed intuitively, based on a presumed picture of pore shape and size. For regular structures (nanotubes, slit-like pores, etc.) mobile adsorption may be more appropriate, while for irregular or random pores localized adsorption mechanism seems to be closer to a physical reality. Nevertheless, even in relatively regular structures there are local irregularities (rows, niches, cones, etc.). The question is, how far such places affect the adsorbate molecule behavior near the solid surface and in what extent the local shape of the surface affects the particle movement making it closer to the localized adsorption mechanism.

This paper attempts to answer such questions by computer analysis of adsorptive molecule movement constraints, due to the surface attractive forces. In particular, a shape and volume of the space occupied by the continuously moving molecule mass center are investigated. Subsequently, conditions for the localized and other adsorption mechanisms are outlined, when compared to the liquid bulk phase properties. To this aim, kinematic equilibrium of individual adsorbate particle near the adsorbent wall is taken under considerations, and then examined in contrast with thermodynamic equilibrium. The adsorbent surface is represented by a set of carbon atoms forming predefined graphite-like nanostructure, and hence included geometrical heterogeneity generates attractive adsorption sites (e.g. nanocone, nanocubes). Such calculations enable 
to examine the effects of particular surface geometry on the shape and volume of adsorbed particles, and so identify a temperature conditions for particular adsorption mechanism domination. The results of these investigations may be useful in selection of the most appropriate mathematical adsorption model, for examination of real porous structures based on adsorption measurements. Hence, it makes it possible to evaluate the porous structure properties more reliably.

\section{Theoretical basis}

\subsection{Kinematics and Maxwell-Boltzmann averaging}

Let us consider a movement of an individual particle of a volatile phase (adsorptive molecule) near an adsorbent solid wall, in time intervals $t_{\text {int }}$ between collisions with other adsorptive molecules. The averaged, in time $t$, molecule movement is limited by an equipotential boundary surface $U_{b d r y}(t)$. On this surface a kinetic energy of two degrees of freedom $k_{B} \tau(t)$ is compensated by a corresponding decrease in an average solid-fluid potential $U_{\text {avge }}(t)$. Thus, the equilibrium of the molecule movement may be described kinematically by the following formulae:

$t_{\text {int }} U_{b d r y}-\int_{0}^{t_{\text {int }}} U_{\text {avge }}(t) d t-k_{B} \int_{0}^{t_{\text {int }}} \tau(t) d t=0$

where $k_{B}$ stands for a Boltzmann constant.

Let us assume that the mass center of this particle moves within a space of volume $V_{f i}$ (free space) and may be found at any phase-space variables $v$ with the same probability (i.e. $v \leq V_{f i}$ ). Hence, time averages $t$ over the particle movement after a suitable lapse of time, frequently longer than $t_{\text {int }}$, correspond to macro scale ensemble averages and the volume in space is preserved, see (Hansen and McDonald 2006). Thereby, kinetic energy $k_{B} \tau(t)$ averaged in time may be equivalently represented by an energy $k_{B} \tau_{i}$ averaged over the movement space in volume $v$. Thus, the kinetic energy is expressed in units $[\mathrm{K}]$ and $\tau_{i}$ denotes an average particle "temperature":

$\tau_{i}=\frac{1}{V_{f i}} \int_{0}^{V_{f i}} \tau(v) d v$

Subsequently, the average solid-fluid potential $U_{\text {avge }}(t)$ and boundary potential $U_{b d r y}(t)$ may be equivalently described by analogous formulas leading to the $U_{\text {avge }}\left(V_{f i}\right)$ and $U_{b d r y}\left(V_{f i}\right)$.

At the boundary, when considering individual particle, the potential is generated by the solid-fluid adhesive forces producing component $U_{b d r y}\left(V_{f}\right)$. Nevertheless, when considering the macro scale, at the boundary, potential is also generated by possible collisions of adsorptive particles in the volatile phase and depends on an external pressure $p$ (i.e. expressed as a $p V$ potential).

Total effects of interactions of the adsorbate particle with volatile phase, represented by the pressure $p$, may be partitioned into subsystems using a collision probability $s_{p i} / s_{p}$ of an individual $i$-th particle. Kinematically, interactions with other particles represented by the $p V$ potential may be related to the free volume $V_{f i}$. Hence, the interaction force $p \cdot s_{p i}$ should be recalculated to an effective pressure $p_{f i}$, and then multiplied by the volume $V_{f}$ :

$p_{f i}=\frac{s_{p i}}{s_{f i}} p, \quad$ and $\quad p V \rightarrow \sum_{i=1}^{N} \frac{s_{p i}}{s_{f i}} V_{f i} p$,

where $s_{p i}$ is an open surface, limiting the particle movement in volume $V_{p i}$ and corresponding to the open surface $s_{f}$, limiting the particle mass center movement in volume $V_{f i}$. For a convenience, the relationship $s_{f i} / V_{f i}$ may be denoted as a specific surface area of the space of particles mass center $a_{f i}$.

Hence, the kinematic equilibrium of the $i$-th particle takes the form:

$p \frac{s_{p i}}{a_{f i}}+U_{b d r y}\left(V_{f i}\right)-U_{\text {avge }}\left(V_{f i}\right)-k_{B} \tau_{i}=0$

The values for the particle volume $V_{p i}$ and the surface ratio $s_{p i} / s_{f i}$ may be roughly evaluated by replacing the real free space of the adsorbate molecule with the sphere of the same volume $V_{f i}$ and corresponding diameter $d_{f i}$. Then the volume $V_{p i}$ is calculated as a sphere with the diameter $d_{p i}$, increased by the adsorbate hard sphere core diameter $\sigma_{f f}$ :

$$
\begin{gathered}
d_{p i}=d_{f i}+\sigma_{f f}, \quad d_{f i} \stackrel{\text { def }}{=}\left(\frac{6}{\pi} V_{f i}\right)^{\frac{1}{3}}, \quad V_{p i}=\frac{\pi}{6} d_{p i}^{3}, \\
\frac{s_{p i}}{s_{f i}}=\left(\frac{d_{p i}}{d_{f i}}\right)^{2}
\end{gathered}
$$

In our study solid is represented by a set of carbon atoms spaced like in a graphite sheet and forming a nanostructure of a predefined shape (nanotube, nanocone or nanocubic niche etc.). Hence, the carbon-fluid potential at a given $k$-th position of adsorbate molecule mass center is calculated by summing up the $\mathrm{LJ}_{12-6}$ potential $u_{C-f k}$ (Lennard-Jones 1931). The latter is performed over all set of carbon atoms forming the solid wall, with a corresponding carbon-fluid intermolecular distance $r_{C-f k}$ :

$u_{C-f_{k}}=4 \varepsilon_{C-f}\left[\left(\frac{\sigma_{C-f}}{r_{C-f_{k}}}\right)^{12}-\left(\frac{\sigma_{C-f}}{r_{C-f_{k}}}\right)^{6}\right]$

where $\varepsilon_{C \text {-fk }}$ and $\sigma_{C \text {-fk }}$ are the potential well-depth and intermolecular diameter, respectively, both calculated 
applying Lorentz-Berthelot (Berthelot 1898; Lorentz 1881) mixing rules from pure-species quantities.

The boundary potential $U_{b d r y}\left(V_{f i}\right)$ is taken arbitrary as a fixed value of $\sum_{C} u_{C-f_{k}}$. Hence, the average potential $U_{\text {avge }}\left(V_{f i}\right)$ is:

$U_{\text {avge }}\left(V_{f i}\right)=\frac{1}{V_{f i}} \int_{0}^{V_{f i}} U_{b d r y}(v) d v$

The values for $V_{f i}$, corresponding to a given $U_{b d r y}$, may be evaluated with analytical or Monte-Carlo calculations. The analytical calculations run initially by searching consecutive points $k$ at an equipotential surface $s_{f i}$ of a given $U_{b d r y}$. If $\sum_{C} u_{C-f_{k}} \approx U_{b d r y}$ the $k$-th point is accepted as belonging to the surface $s_{f i}$ (more precisely, to a volume $\left.\delta s_{f i} \delta \delta r_{C-f k}\right)$. Then, the volume $V_{f i}$ is determined, as a volume of space limited by all points found at the $s_{f}$ surface. Starting with a minimum potential $U_{b d r y}$ for which $V_{f i} \approx 0$, the calculations run step by step with an increasing $U_{b d r y}$ by a small value $\delta U_{b d r y}$. In the Monte-Carlo technique $u_{C-f k}$ is calculated many times for randomly chosen points $k$ (shots). The point $k$ is accepted as an admissible $n_{\text {adm }}$ if respects geometrical constraints of the solid and as a successful $n_{v}$ if additionally fulfills the relation $\sum_{C} u_{C-f_{k}} \leq U_{b d r y}$. Having an appropriately large number of admissible shots $n_{\text {adm }}>10^{6}$ with a number $n_{v}$ of successful shots, one can calculate the volume $V_{f i}$ corresponding to a given $U_{b d r y}: V_{f i} \approx n_{v} / n_{a d m}$. In both techniques the values for $U_{\text {avge }}\left(V_{f i}\right)$ are calculated by numerical integration of the $U_{b d r y}\left(V_{f i}\right)$ - see Eq. (7). Subsequently the corresponding particle volume $V_{p i}$ is calculated according to Eq. (5).

So far as a movement of a single particle is considered only, one should assume that there are no particles in volatile phase, i.e. $p=0$. In this case, having the values for $U_{\text {avge }}\left(V_{p i}\right)$ and $U_{b d r y}\left(V_{p i}\right)$ one can calculate with Eq. (4) the values for $\tau_{i}$ corresponding to a given $V_{p i}$. For a convenience, the quantities in Eq. (4) may be also related to one mole of particles, enabling to calculate the kinematic equilibrium temperature $\tau_{i}$ (corresponding to a given $V_{f i}$, $V_{p i}$, and so to $\left.U_{\text {avge }}\left(V_{p i}\right), U_{b d r y}\left(V_{p i}\right)\right)$ :

$$
\begin{aligned}
\tau_{i} & =\left(\frac{U_{b d r y}\left(V_{p i}\right)-U_{\text {avge }}\left(V_{p i}\right)}{k_{B}}\right) \times \frac{N_{A}}{N_{A}} \\
& =\frac{U_{m}\left(V_{m_{p i}}\right)-U_{m_{\text {adh }}}\left(V_{m_{p i}}\right)}{R}
\end{aligned}
$$

and

$$
\begin{gathered}
V_{m_{f i}} \stackrel{\text { def }}{=} N_{A} V_{f i}, \quad V_{m_{p i}} \stackrel{\text { def }}{=} N_{A} V_{p i}, \\
U_{m}\left(V_{m_{p i}}\right) \stackrel{\text { def }}{=} N_{A} U_{b d r y}\left(V_{m_{p i}}\right), \\
U_{m_{a d h}}\left(V_{m_{p i}} \stackrel{\text { def }}{=} N_{A} U_{\text {avge }}\left(V_{m_{p i}}\right)\right.
\end{gathered}
$$

where $N_{A}$ is an Avogadro constant and $\mathrm{R}$ is a gas constant.

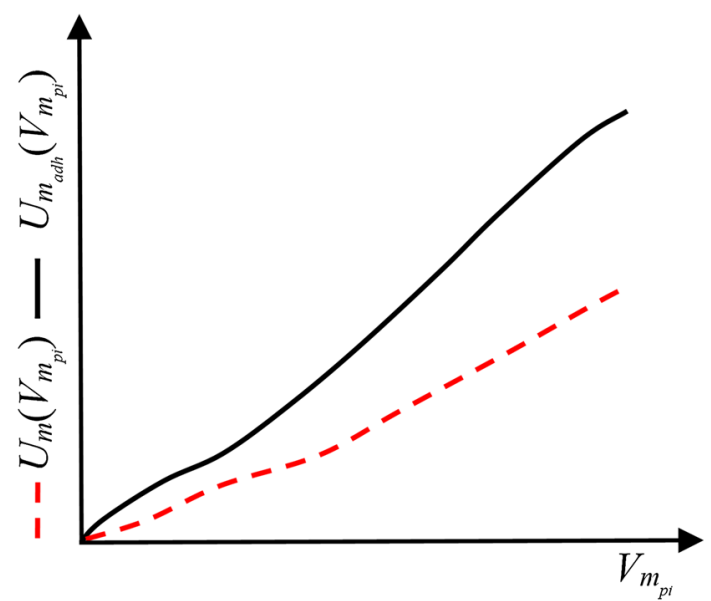

Fig. 1 Schematic profile of solid-fluid potential $U_{\text {madh }}\left(V_{m p i}\right)$ function (dashed line) and boundary solid-fluid potential $U_{m}\left(V_{m p i}\right)$ function (solid line) for an individual particle moving in a space of volume $V_{m p i}$

The functions $U_{m}\left(V_{m p i}\right)$ and $U_{m a d h}\left(V_{m p i}\right)$ are schematically shown in Fig. 1. Subsequently, the inverse relationship $V_{m p i}\left(\tau_{i}\right)$ calculated with Eq. (8) is schematically shown in Fig. 2.

Let us denote $V_{\text {ind }}$ as a maximum volume of a free movement space (e.g. close to an averaged volume of particle movement at a critical point), thus it is not likely that this space may be shared with other molecules. The corresponding temperature $\tau_{\text {ind }}$ is found with Eq. (8) and $\tau_{\text {ind }}=\arg \left\{\min \left\{V_{\text {mpi }}\left(\tau_{\text {ind }}\right)=V_{\text {ind }}\right\}\right\}$. When the particle temperature is appropriately low, i.e. $\tau_{i} \leq \tau_{\text {ind }}$ and so $V_{m p i}\left(\tau_{i}\right) \leq V_{\text {ind }}$, one may assume that it occupies a localized site, so the localized monolayer adsorption takes place. If the temperature is $\tau_{i}>\tau_{\text {ind }}$, but is still

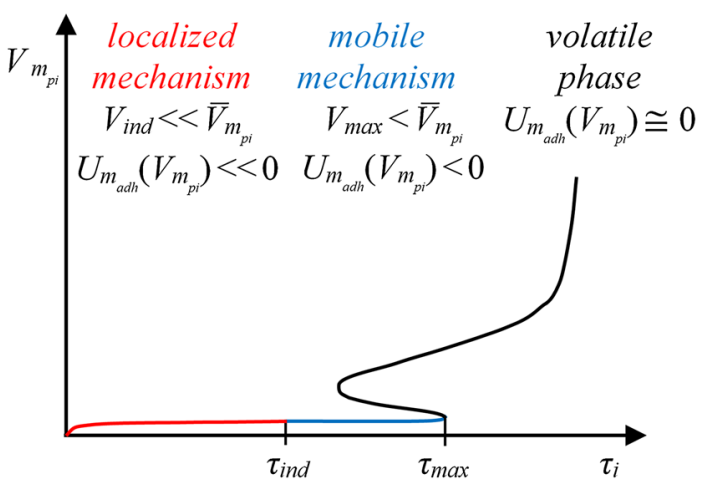

Fig. 2 Hypothetical $V_{m p i}\left(\tau_{i}\right)$ relationship for one mole of individual particles moving in the free volume space $V_{m f i}\left(\tau_{i}\right)$ calculated with Eq. (8). $V_{\text {ind }}$ stands for the maximum volume $V_{m p i}\left(\tau_{i}\right)$ of one mole of individual particles, at the temperature $\tau_{\text {ind }}$. $V_{\max }$ stands for the maximum occupied volume at the individual spaces $V_{m p i}\left(\tau_{i}\right)$ or shared volume of space $V_{\text {mpi }}\left(\tau_{i}\right)$ with $j$ molecules, at the temperature $\tau_{\max }$. The intervals $0<\tau_{i} \leq \tau_{\text {ind }}$ and $\tau_{\text {ind }}<\tau_{i} \leq \tau_{\max }$ corresponds to the localized or possible mobile mechanisms, respectively 
appropriately low, that the particle may be captured into an interaction potential well, one may assume that the particle may easily occupy an individual spaces $V_{m p i}\left(\tau_{i}\right)$ or joint a space $V_{m p i}\left(\tau_{i}\right)$ containing $j$ molecules. Thus, maximum volume occupied by individual particles $j$ (depending on pressure $p$ ) may be expressed in general form $V_{\max }=$ $V_{m p i}\left(\tau_{i}\right) \cdot j$, and so the corresponding maximum temperature $\tau_{\max }=\arg \left\{V_{\text {mpi }}\left(\tau_{i}\right)=V_{\max } \gg V_{\text {ind }}\right\}$. If the space of volume $V_{\max }$ covers a large fraction of the solid surface, adsorption mechanism may be seen as a purely mobile, otherwise it may be closer to the clustering based or to locally mobile adsorption (based on forming a local 2D fluid). Nevertheless, the derived formulas are legitimate only for characterization of localized adsorption mechanism properties. Thus they don't decide on the mechanism in the temperature range $\tau_{\text {ind }}<\tau_{i} \leq \tau_{\max }$ (i.e. it may be mobile or clustering based mechanism). Finally, particles of the temperature $\tau_{i}>\tau_{\max }$ are treated as belonging to the volatile phase (not adsorbed).

While accepting the abovementioned criteria, one should consider that the total volume $V_{m p i}$ and potentials $U_{\text {madh }}\left(V_{m p i}\right), U_{m}\left(V_{m p i}\right)$ are additive at appropriately low temperature range $\tau_{i} \leq \tau_{\text {ind }}$. Moreover, excess energy evolved due to the interaction of the captured particle with solid wall is totally withdrawn to keep the constant temperature $T$ in isothermal adsorption. Hence, the quantities in Eq. (4) may be averaged over all set of molecules (e.g. containing $N$ particles) with Maxwell-Boltzmann distribution function $f\left(\tau_{i}, T\right)$ at a given temperature $T$. If the individual particles temperature is $\tau_{\text {ind }}<\tau_{i} \leq \tau_{\max }$, the additivity of the total volume of adsorbed individual particles is no longer valid. Nevertheless, for a consistency of the proposed description it is useful to evaluate the number of particles $j$ sharing occupied volume also in the range $V_{\text {ind }}<V_{\text {mpi }}\left(\tau_{i}\right) \leq V_{\max }$. Assuming, that the volume $V_{\text {ind }}$ corresponds to the maximum separated volume of one mole of individual particles, the upper bound for a volume $V_{m p i}\left(\tau_{i}\right)$ of the number of particles, may be evaluated with simple expression $j=V_{m p i}\left(\tau_{i}\right) / V_{\text {ind }}$. As well, the potentials $U_{\text {madh }}\left(V_{m p i}\right)$ and $U_{m}\left(V_{m p i}\right)$ may be seen as proportionally shared in terms of the number of $j$ particles. Thus, the following expressions are valid:

$$
\begin{gathered}
j=\frac{V_{m_{p i}}}{V_{\text {ind }}}, \quad U_{m}\left(V_{m_{p i}}\right)=\frac{U_{m}\left(V_{m_{p i}}>V_{\text {ind }}\right)}{j}, \\
U_{m_{a d h}}\left(V_{m_{p i}}\right)=\frac{U_{m_{a d h}}\left(V_{m_{p i}}>V_{i n d}\right)}{j}
\end{gathered}
$$

Hence, it enables for the averaging of the quantities in Eq. (4) also up to the limit $\tau_{i}=\tau_{\max }$.

The integration of the distribution function $f\left(\tau_{i}, T\right)$ up to the limits $\tau_{\text {ind }}$ and $\tau_{\max }$ leads to the fraction $w_{\text {ind }}$ and $w_{\max }$ of volatile phase particles, at the particular temperature $T$. Following adsorption mechanism identification criteria

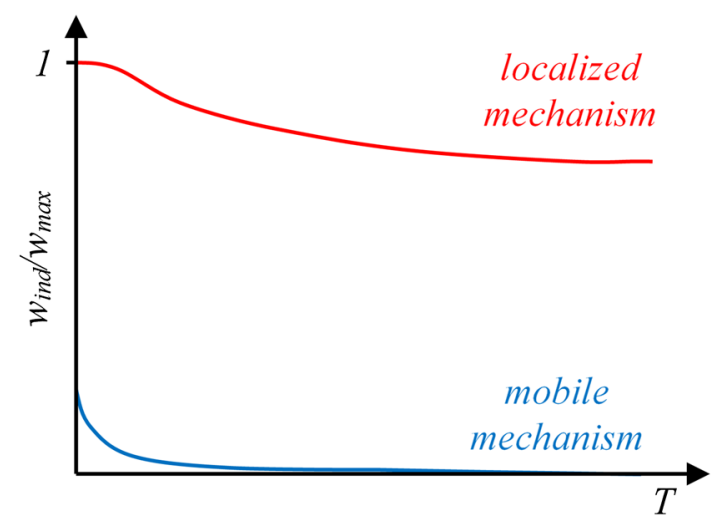

Fig. 3 Hypothetical relationship for $w_{\text {ind }} / w_{\max }$ ratio as a function of temperature $T$, describing the statistical significance of the localized and possible mobile mechanisms in real adsorption system

$w_{\text {ind }}$ is a localized mechanism fraction and so $w_{\max }$ correspond to the other mechanisms, e.g. possible mobile mechanism (see Fig. 3):

$w_{\text {ind }} \stackrel{\text { def }}{=} \int_{0}^{\tau_{\text {ind }}} f\left(\tau_{i}, T\right) d \tau_{i}, \quad w_{\text {max }} \stackrel{\text { def }}{=} \int_{0}^{\tau_{\text {max }}} f\left(\tau_{i}, T\right) d \tau_{i}$

where the remaining part $\left(1-w_{\max }\right)$ is the fraction of one mole of individual particles of volatile phase $\pi$. The values obtained in Eq. (11) refer to the idealized adsorptive volatile phase fractions that may be adsorbed with particular mechanism, but it does not specify liquidlike phase particles properties.

At the intervals between collisions, each $i$-th particle in the adsorption system is at the kinematic equilibrium and the average temperature of adsorbed molecules is expressed as:

$T_{k} \stackrel{\text { def }}{=} \frac{1}{N_{\text {ind }}} \sum_{i=1}^{N_{\text {ind }}} \tau_{i}=\frac{1}{w_{\text {ind }}} \sum_{n=1}^{N_{\text {ind }}} f\left(\tau_{n}, T\right) \tau_{n} \Delta \tau_{n}$

where $N_{\text {ind }}$ is a number of particles corresponding to the particular temperature $\tau_{i}$, fulfilling the constraint $\tau_{i} \leq \tau_{\text {ind }}$.

Hence, by virtue of Eqs. (8) and (12) the following equation is valid:

$$
\begin{aligned}
T_{k} & =\frac{1}{w_{\text {ind }} R} \sum_{n=1}^{N_{\text {ind }}} f\left(\tau_{n}, T\right)\left(p \frac{s_{p n}}{a_{m_{\text {fn }}}}+U_{m_{n}}\left(V_{m_{p n}}\right)-U_{m_{\text {all } h} n}\left(V_{m_{p n}}\right)+\right) \Delta \tau_{n} \\
& =\frac{1}{R}\left(p\left\langle\frac{s_{p n}}{a_{m_{m_{n}}}}\right\rangle+\bar{U}_{m}\left(\bar{V}_{m_{p}}\right)-\bar{U}_{m_{a d h}}\left(\bar{V}_{m_{p}}\right)\right)
\end{aligned}
$$

where ${ }^{-}$refers to the quantities at the given temperature $T_{k}$, averaged over the interval $\tau_{i} \in\left(0 ; \tau_{\text {ind }}\right)$. The averaging is performed for all quantities in analogy to the following formulae:

$\bar{V}_{m_{p}} \stackrel{\text { def }}{=} \frac{1}{N_{\text {ind }}} \sum_{i=1}^{N_{\text {ind }}} V_{m_{p i}}=\sum_{n=1}^{N} f\left(\tau_{n}, T\right) V_{m_{p n}} \Delta \tau_{n}$ 
Moreover, Eq. (13) implies that relationship $V_{m p i}\left(T_{k}\right.$, $p=0$ ) may be seen as the upper bound for a volume $V_{m p i}\left(T_{k}, p\right)$. It may be easily examined by solving nonlinear system, of Eq. (13) and equilibrium vapor pressure estimation with quasipolynomial Riedel equation (Vetere 1991). Thus, enabling to find corresponding temperature $T_{k}$ at the pressure $p$ :

$\ln p=A \ln T_{k}+\frac{B}{T_{k}}+C+D T_{k}^{2}$

where $p$ is expressed in units $[\mathrm{kPa}]$ and $A, B, C, D$ are empirical constants that may be taken from KDB (CHERIC 2015; Kang et al. 2001).

\subsection{Thermodynamics}

Let us assume that $\tau_{\text {ind }}=\infty$ and adsorption system, held at a constant temperature $T$, consists of $N_{\text {ind }}$ separated subsystems, each containing one molecule $i$. The volume of any molecular system at its equilibrium state minimizes free enthalpy $G$ of the system:

$\left.\frac{\partial G}{\partial V_{p}}\right|_{T}=0, \quad G=U_{a d s}+p V_{p}-T S_{a d s}$

where $U_{a d s}, S_{a d s}, V_{p}$ denote the total energy, entropy and volume of adsorbate molecules, respectively.

For such a system internal entropy and energy are additive, so the following equations are valid:

$$
\begin{gathered}
S_{a d s}=S_{\text {conf }}+\sum_{i=1}^{N_{\text {ind }}} S_{i}, \quad S_{i}=-k_{B} \ln \frac{\delta V}{V_{f i}}=k_{B} \ln V_{f i}+S_{0}, \\
U_{a d s}=\sum_{i=1}^{N_{\text {ind }}} U_{\text {avge }}\left(V_{f i}\right)
\end{gathered}
$$

where $S_{\text {conf }}$ denotes the configurational entropy of the system, $S_{i}$ stands for the $i$-th particle internal entropy (it was assumed that finding of an individual particle in any infinitesimal volume $\delta V$ is equally probable), $S_{O}$ is a constant of no importance and $U_{\text {avge }}\left(V_{f i}\right)$ is defined in Eq. (7).

The configurational entropy does not depend on adsorbate molecules volume, so $\partial S_{\text {conf }} / \partial V_{p}=0$. In turn, effects of any change in free volume $V_{f i}$ of an $i$-th particle are limited to this particle internal entropy and temperature. Hence, the entropy term and its total differential should be written in the following form:

$$
\begin{gathered}
T S_{a d s}=T S_{\text {conf }}+T \sum_{i=1}^{N_{\text {ind }}} S_{i}, \\
\frac{\partial}{\partial V_{p}}\left(T S_{a d s}\right)=T \sum_{i=1}^{N_{\text {ind }}} \frac{\partial S_{i}}{\partial V_{p}}=T \sum_{i=1}^{N_{\text {ind }}} \frac{\partial S_{i}}{\partial V_{f i}} \frac{\partial V_{f i}}{\partial V_{p}}
\end{gathered}
$$

Thus, by virtue of Eqs. (7), (17) and (18), free enthalpy total differential may be expressed:

$$
\begin{aligned}
\left.\frac{\partial G}{\partial V_{p}}\right|_{T} & =\left.\sum_{i=1}^{N_{\text {ind }}} \frac{\partial V_{f i}}{\partial V_{p}} \frac{\partial G_{i}}{\partial V_{f i}}\right|_{T} \\
& =p+\sum_{i=1}^{N_{\text {ind }}} \frac{\partial V_{f i}}{\partial V_{p}} \frac{1}{V_{f i}}\left(U_{b d r y}\left(V_{f i}\right)-U_{\text {avg }}\left(V_{f i}\right)-k_{B} T\right)=0
\end{aligned}
$$

Let us take that any change in the total volume $V_{p}$ splits into the volumes $V_{p i}$ and $V_{f i}$ in proportion to the surfaces $s_{p}$, $s_{p i}$ and $s_{f i}$ of corresponding spaces (i.e. any change in volume may be expressed by small change $d r$ ). Hence, the differentials fulfill the relations:

$\frac{\partial V_{f i}}{\partial V_{p}}=\frac{s_{f i}}{s_{p}}, \frac{\partial V_{p i}}{\partial V_{p}}=\frac{s_{p i}}{s_{p}}, \quad$ where $s_{p}=\sum_{i=1}^{N_{\text {ind }}} s_{p i}$

Moreover, the total effect of interactions of adsorbate particles $N_{\text {ind }}$ with volatile phase, represented in Eq. (19) by the pressure $p$, may be partitioned onto the subsystems using the collision probability $s_{p i} / s_{p}$ of an individual $i$-th particle:

$p=\sum_{i=1}^{N_{\text {ind }}} \frac{s_{p i}}{s_{p}} p$

For a convenience, let us again relate the quantities obtained in Eq. (19) to one mole of particles according to Eq. (9). By virtue of Eqs. (20) and (21) into Eq. (19) the thermodynamic equilibrium may be expressed in the following form:

$$
\begin{aligned}
& \left(\sum_{i=1}^{N_{\text {ind }}} s_{p i} p+\frac{1}{N_{\text {ind }}} \sum_{i=1}^{N_{\text {ind }}} a_{f i} U_{b d r y}\left(V_{f i}\right)-\frac{1}{N_{\text {ind }}} \sum_{i=1}^{N_{\text {ind }}} a_{f i} U_{\text {avge }}\left(V_{f i}\right)\right. \\
& \left.-\frac{1}{N_{\text {ind }}} \sum_{i=1}^{N_{\text {ind }}} a_{f i} k_{B} T\right) \times\left(\frac{N_{A}}{N_{A}}\right)^{2}=0
\end{aligned}
$$

Subsequently, taking into account quantities defined in Eq. (9), the thermodynamic equilibrium temperature $T_{t}$ may be obtained:

$T_{t}=\frac{1}{R} \frac{\sum_{i=1}^{N_{\text {ind }}}\left(p s_{p i}+a_{m_{f i}} U_{m}\left(V_{m_{p i}}\right)-a_{m_{f i}} U_{m_{a d h}}\left(V_{m_{p i}}\right)\right)}{\sum_{i=1}^{N_{\text {ind }}} a_{m_{f i}}}$

In order to evaluate the adequacy of kinematic considerations to the analysis of adsorbed particles properties, let us calculate the thermodynamic temperature $T_{t}$. Thus, let us assume that the distributions of the quantities $U_{\text {madh }}\left(V_{m p i}\right)$ and $U_{m}\left(V_{m p i}\right)$ and corresponding $s_{p i}$ and $a_{m f i}$ is the same as 
in the kinematic equilibrium, at the temperature $T_{k}$ (see Eq. (13)):

$$
=\frac{1}{R} \frac{\sum_{n=1}^{N} f\left(\tau_{n}, T\right) a_{m_{f n}}\left(p \frac{s_{p n}}{a_{m_{f n}}}+U_{m_{n}}\left(V_{m_{p n}}\right)-U_{m_{\text {adl }, n}}\left(V_{m_{p n}}\right)\right) \Delta \tau_{n}}{\sum_{n=1}^{N}\left(\tau_{n}, T\right) a_{m_{f n}} \Delta \tau_{n}}
$$

where $N$ stands for the number of the available $\tau_{n}$, found with Eq. (8).

The relationship $V_{m p}\left(T_{t}\right)$ may be viewed as a realistic description of adsorbate volume in real localized adsorption systems. Moreover, the comparison of Eqs. (24) and (13) provides information that the particle temperature distribution in real systems is the Maxwell-Boltzmann distribution, modified with $a_{m f n}$ weights.

\subsection{Adsorption mechanism domination}

In real adsorption system, it is not likely that adsorption will take place only with purely localized or purely mobile mechanism. In turn, intuitively both mechanisms are expected, even if single heterogeneity is considered as a model (e.g. cavity, corner). Hence, the particular mechanism domination may be evaluated based on the molecules fractions $w_{\text {ind }}$ and $w_{\max }$, see Eq. (11). One may consider the ratio of the obtained fractions, as follows:

$$
\begin{aligned}
& \frac{w_{\text {ind }}}{w_{\text {max }}} \stackrel{d e f}{\approx} 1 \text { for purely localized mechanism, } \\
& \frac{w_{\text {ind }}}{w_{\text {max }}} \stackrel{\text { def }}{\approx} 0 \text { for purely mobile mechanism }
\end{aligned}
$$

The relationships in Eq. (25) are exceptional cases and most likely in real adsorption systems they might not be observed. Nevertheless, at the given temperature $T$ of experimental adsorption measurements, it is possible to select the most appropriate mathematical adsorption model based on $w_{\text {ind }} / w_{\max }$ ratio (see Fig. 3).

The localized mechanism in uniBET model, elaborated in our team, may be considered as a mixing of molecules being in the reference liquidlike state, with cells placed at the adsorbent surface, see e.g.: (Duda et al. 2013; Milewska-Duda et al. 2000; Milewska-Duda and Duda 2001). In such a view a key parameter is an effective adhesion energy $E_{a d h}$, related to a measurable monolayer adsorption energy $Q_{A}$ with a Huggins formula (Flory 1953):

$Q_{A}=E_{\text {vap }}-\zeta E_{a d h}$

where $E_{\text {vap }}$ is the cohesion (evaporation) energy of the adsorbate in its reference state and $\zeta$ is a surface texture parameter describing possible contact of adsorbate with neighboring adsorbent molecules (intermolecular effective contact ratio). Adhesion energy $E_{a d h}$ is evaluated for a pair of molecules being in a full contact, by combining, via Berthelot rule, adsorbate and adsorbent cohesion energies:

$E_{\text {adh }}=2 \delta_{c} \sqrt{E_{\text {vap }} V_{m}}$

where $\delta_{c}$ is a solubility parameter and $E_{\text {vap }}$ is evaluated with $P V T$ relationship for $V_{m}$, a molar volume of adsorbate in its reference state (for vaporous substances it is the liquid state), see (Milewska-Duda and Duda 2009).

In order to get an insight into adequacy of the kinematic evaluations in Eq. (13) one may calculate the $\zeta$ parameter. Thus, taking the values for $E_{a d h}\left(V_{m p}\right)$ found with Eq. (27), $V_{m p}$ and corresponding effective adhesion energy $U_{\text {madh }}$ $\left(V_{m p}\right)$ calculated with Eq. (10) and averaged with Eq. (14):

$\zeta=\frac{\bar{U}_{m_{a d h}}\left(\bar{V}_{m_{p}}\right)}{\bar{E}_{a d h}\left(\bar{V}_{m_{p}}\right)}$

Despite the simplifications involved in Eq. (10) for the volume $V_{\text {ind }}<V_{m p i}<V_{\text {ind }}$, the parameter $\zeta$ found with Eq. (28) may be viewed as a basis for validation of the proposed approach, when compared to empirical values published e.g. in (Duda et al. 2013).

\section{Results and discussion}

Accuracy of the proposed description was evaluated performing the calculations for interaction of nitrogen with carbon atoms of materials surface. The modeled materials were open carbon nanocone (CNC), graphite sample (GS) with a defect in a form of a cavity, soft activated carbon (AC) composed of graphite crystallites and capped singlewalled carbon nanotube (SWCNT). Commonly, an adsorbent structure is modeled as continuous carbon surface, e.g. in DFT formalism. However, in our view more detailed discreet distribution of carbon atoms may be of significance. Hence, it was assumed that the surface consists of carbon atoms spaced like in a graphene and forming a structure of a predefined shape, thus also a geometrical heterogeneity. In particular, CNC model is composed of the wrapped graphene sheet, respecting geometrical requirements for a seamless connection. The cone height is $4.0 \mathrm{~nm}$ and a base is $2.5 \mathrm{~nm}$ diameter with a disclination angle $240^{\circ}$. The cone itself may be seen as a geometrical heterogeneity. SWCNT model was constructed in the similar manner, and so the graphene layer is wrapped yielding armchair nanotube of $4.0 \mathrm{~nm}$ diameter. In order to produce geometrical heterogeneity the tube was capped, so the total length is $6.0 \mathrm{~nm}$. Due to a steric constraints cap is built of a 5-, 6- and 7-membered carbocycles, see (Robinson and Marks 2014) for more details. In turn, GS 


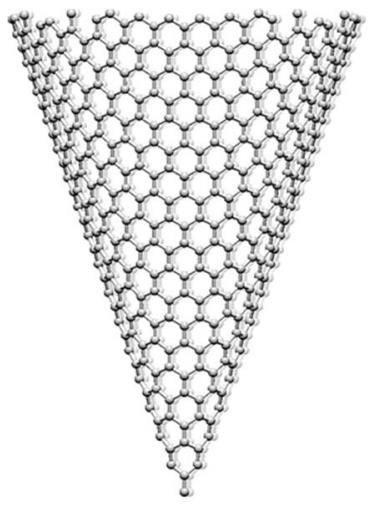

Fig. 4 Adsorbent models (model parameters are given in brackets, all distances are measured between the center of carbon atoms), from left: open $\mathrm{CNC}$ of $4.0 \mathrm{~nm}$ height and $2.5 \mathrm{~nm}$ diameter; armchair, one- side capped SWCNT of $6.0 \mathrm{~nm}$ height and $4.0 \mathrm{~nm}$ diameter, sixlayers GS with a cavity at the two top layers, soft AC consisting of six microcrystallites, respectively

Table 2 Calculated adsorption mechanism (localized versus other) identification parameters

\begin{tabular}{lcccc}
\hline & AC & CNC & GS & SWCNT \\
\hline$\tau_{\text {ind }}(\mathrm{K})$ & 171.4 & 262.5 & 260.1 & 4.5 \\
$\tau_{\max }(\mathrm{K})$ & 203.6 & 277.8 & 373.7 & 158.7 \\
$V_{\max }\left(\mathrm{cm}^{3} / \mathrm{mol}\right)$ & 130.8 & 132.5 & 127.1 & 370.3 \\
$T_{k}\left(V_{c}, p=0\right)(\mathrm{K})$ & 83.7 & 114.7 & 111.2 & 2.6 \\
$T_{k}\left(V_{c}, p\right)(\mathrm{K})$ & 83.7 & 114.7 & 111.2 & 2.6 \\
$T_{t}\left(V_{c}, p\right)(\mathrm{K})$ & 96.8 & 135.2 & 137.4 & 3.1 \\
$\zeta_{\text {const }}$ & 0.82 & 0.65 & 0.51 & 0.36 \\
$\frac{w_{\text {ind }} \text { a }}{w_{\text {max }}}$ & 0.99 & 0.92 & 0.96 & 0.01
\end{tabular}

a The values for $w_{\text {ind }}$ and $w_{\max }$ ratio are given for nitrogen normal boiling temperature $T_{B}$ (the temperature of typical adsorption measurements)

It was expected that structural diversity of $\mathrm{AC}, \mathrm{CNC}$ and GS materials would generate energetical heterogeneity, so that a depth of a potential well $U_{m}\left(V_{m p i}\right)$ would be deep enough to produce a temperature $\tau_{i}<\tau_{\text {ind }}$ (see Fig. 2). The results presented in Figs. $5 \mathrm{a}-\mathrm{c}$ and $6 \mathrm{a}-\mathrm{c}$ confirms this expected feature, while referring to Fig. 2. Thus, maximum
Table 1 Calculation parameters used in the study

\begin{tabular}{llllllllll}
\hline & $\sigma(\mathrm{nm})$ & $\varepsilon / k_{B}(\mathrm{~K})$ & $T_{c}(\mathrm{~K})$ & $T_{B}(\mathrm{~K})$ & $V_{c}\left(\mathrm{~cm}^{3} / \mathrm{mol}\right)$ & $A$ & $B$ & $C$ & $D$ \\
\hline $\mathrm{N}-\mathrm{N}$ & 0.36 & 71.4 & 126.2 & 77.8 & 89.8 & -4.5 & -976.0 & 36.3 & $8.36 \cdot 10^{-5}$ \\
$\mathrm{C}-\mathrm{C}$ & $0.34^{\mathrm{a}, \mathrm{b}}$ & $28.0^{\mathrm{a}}$ & - & - & - & - & - & - & - \\
& $0.35^{\mathrm{c}}$ & $30.6^{\mathrm{b}}$ & & & & & & & \\
& & $35.9^{\mathrm{c}}$ & & & & & & &
\end{tabular}

\footnotetext{
$\mathrm{AC}$ and GS models

${ }^{b} \mathrm{CNC}$ model

c SWCNT model
}

Lennard-Jones pure species parameters for: nitrogen (Bird and Stewart 2001), AC and GS models (Siderius and Gelb 2011), CNC model (Baowan and Thamwattana 2011) and SWCNT model (Kaukonen et al. 2012). Riedel equation parameters (CHERIC 2015). Remaining nitrogen parameters (Reid et al. 1987) 


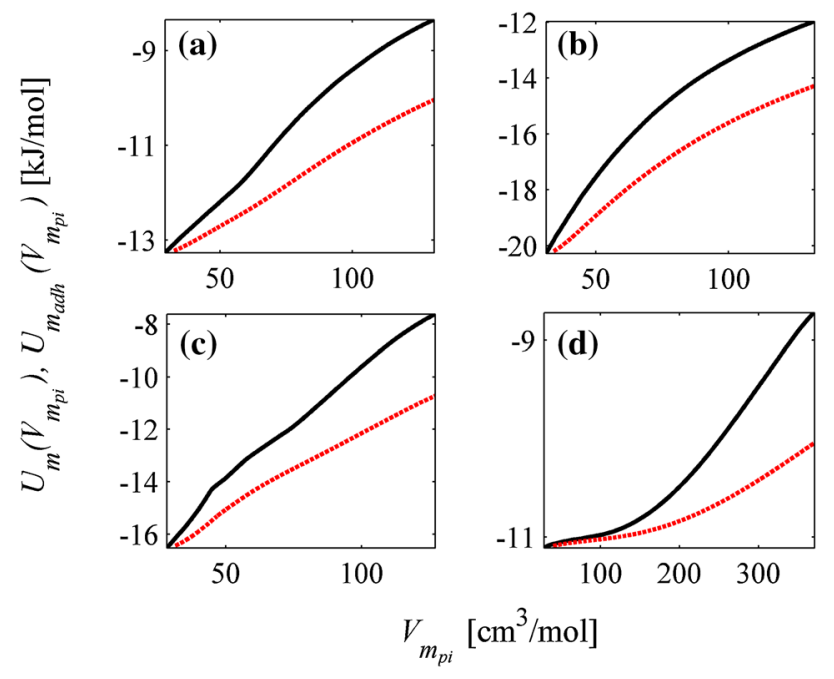

Fig. 5 Interaction potential $U_{m}\left(V_{m p i}\right)$ (solid line) and adhesion energy $U_{\text {madh }}\left(V_{m p i}\right)$ (dashed line) for: a $\mathrm{AC}, \mathbf{b} \mathrm{CNC}, \mathbf{c} \mathrm{GS}$ and d SWCNT models

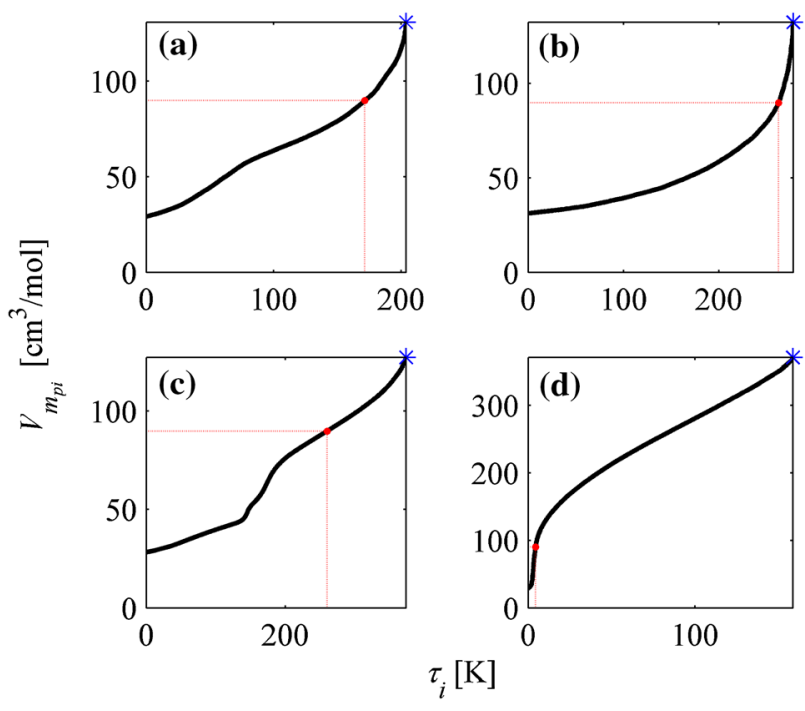

Fig. 6 Calculated volume $V_{m p i}$ and corresponding temperature $\tau_{i}$ for: a AC, b CNC, c GS and d SWCNT models. Dot corresponds to the values of $\tau_{\text {ind }}$ and $V_{\text {ind }}$, and asterisk to the $\tau_{\max }$ and $V_{\max }$

individual volume $V_{\text {ind }}=V_{c}$ was taken as a reasonable value, and then the corresponding temperature $\tau_{\text {ind }}$ was identified.

Moreover, in case of CNC model it was intuitively expected that geometrical heterogeneity will produce only one adsorption site, i.e. at the cone top. This may be observed in low range of potentials in Fig. $7 \mathrm{a}$ and $\mathrm{g}$, where equipotential lines are continuous and individual. In turn, for GS (Fig. 7b, e) and AC (Fig. 7c, f) materials due to the structural heterogeneity of the cavity, and microcrystallites distribution, more than one separated active site was expected. This may be observed at the equipotential surface cross-sections, where more than one local minimum of separated volume were identified. It should be also noticed in Fig. 7 that despite a sufficiently large number of successful shots in Monte-Carlo method, the discontinuities and slight differences in the minimum values of the potential, may be observed. This is due to interpolation of random, scattered configurations $v$ in order to determine an equipotential surface, and may be easily eliminated in analytical calculations. Anyway, the results quality of the Monte-Carlo method is good enough to perform these analyses.

What concerns an identification of the other mechanism (e.g. possible mobile mechanism), it was found out there may be observed a temperature range, wherein two volume values correspond to one value of the temperature (see Fig. 2). It indicates coexistence of two phases: dilute liquidlike and bulk, and also facilitates a determination of a temperature $\tau_{\max }$. Thus, the latter was identified as a corresponding to the maximum volume of dilute liquidlike phase $V_{\max }$, for the phases coexistence region.

SWCNT material was designed as a model with relatively small expected energetical heterogeneity (at the nanotube cap), resulting in adsorbate condensation not exceeding the critical volume (Zhao et al. 2001). Accordingly, the highest minimum value of the potential $U_{m}\left(V_{m p i}\right)$ was obtained for SWCNT (Fig. 5d), and so the structural diversity produces relatively shallow potential well-depth. Qualitatively, it occurs at the equipotential surface crosssections, where indistinct and connected with each other lines may be observed see Fig. $7 \mathrm{~d}$ and $\mathrm{h}$. Therefore, identification of the separated volume $V_{\text {ind }}$ corresponding to the localized adsorption mechanism is ambiguous (see Fig. 6d).

Very interesting results, providing a comparison of the kinematic and thermodynamic temperatures with a liquid nitrogen thermal expansion curve are shown in Fig. 8. They compare the curves corresponding to a given $V_{m p i}$, see Eq. (13) $T_{k}\left(V_{m p i}, p=0\right), T_{k}\left(V_{m p i}, p\right)$ and Eq. (24) $T_{t}\left(V_{m p i}, p\right)$, with the thermal expansion curve found with (Milewska-Duda and Duda 2009). This comparison brings strong confirmation of the adequacy of the proposed approach. Moreover, it provides an information that the pressure effect on the volume is insignificant, when the indistinguishability of the curves $T_{k}\left(V_{m p i}, p=0\right)$ and $T_{k}\left(V_{m p i}, p\right)$ is noticed, (less than $1 \%$ of a difference, see Table 2).

For CNC (Fig. 8b) and GS (Fig. 8c) materials, the $T_{t}$ curves are almost in perfect agreement with calculated nitrogen expansion curve. It means that for such structures the classical localized adsorption assumption (i.e. vaporous adsorbate is in a liquid state) is fully acceptable. Nevertheless, the $T_{t}$ curve for AC (Fig. 8a) seems to suggest that this assumption is valid only in the low temperature range. 
Fig. $7 U_{m}\left(V_{m p i}\right)$ equipotential surface cross-sections, interpolated from random, scattered configurations (colorbar correspond to the values of the potential). Crosssections for: AC model, at the $\mathrm{AC}$ width $2.4 \mathrm{~nm}(\mathbf{b})$ and along AC length (e), CNC model, at the $\mathrm{CNC}$ height $1.3 \mathrm{~nm}$ (a) and along CNC center (g), GS model, at the GS width $1.4 \mathrm{~nm}$ (c) and through the cavity center (f) and for SWCNT model, at the SWCNT height $1.3 \mathrm{~nm}$ (d) and along SWCNT center (h) (Color figure online)

$-18$ $-16$ $-14$ $-12$ $-10$ $-8 \quad-6$ $-4 \quad-2$

(a)

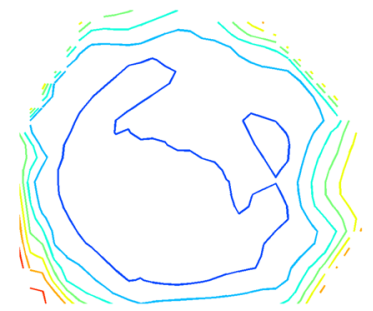

(d)

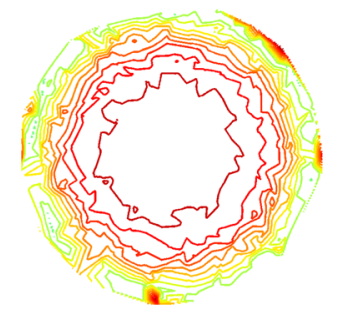

(e)
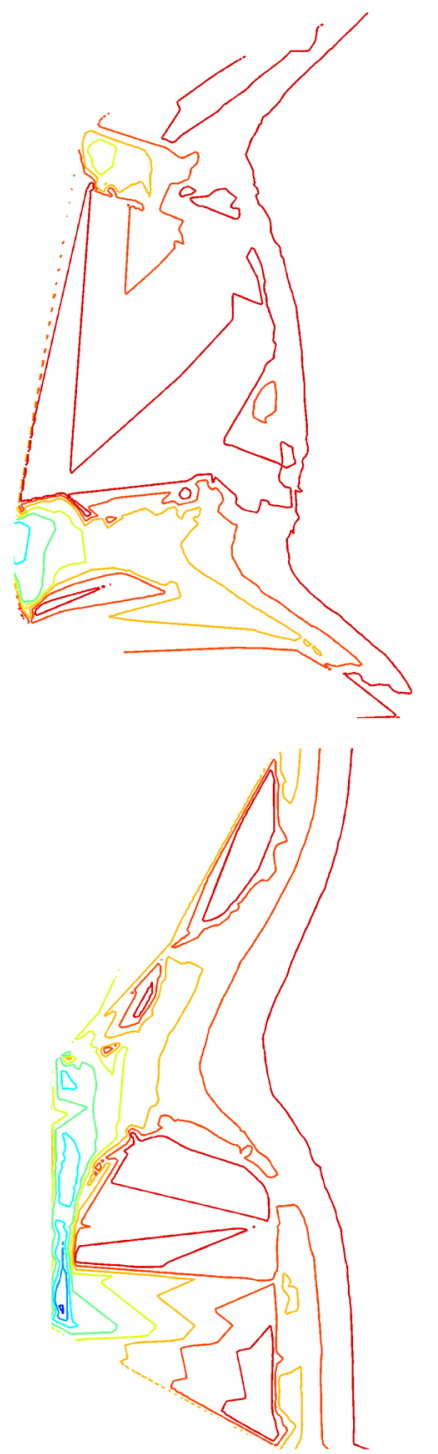

(f)

(b)

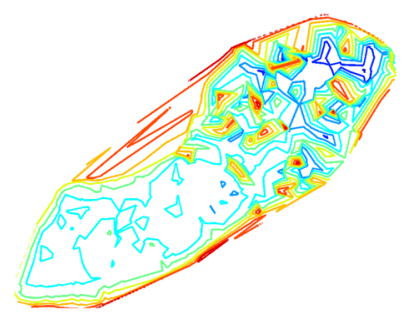

(c)

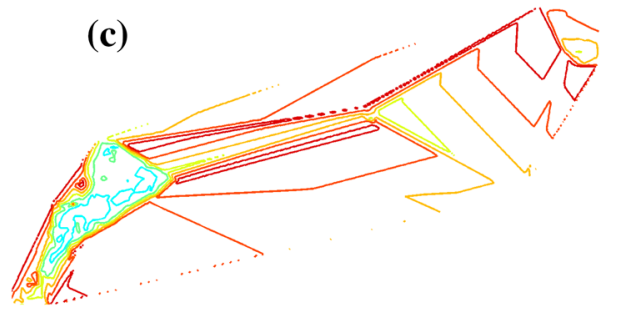

(g)

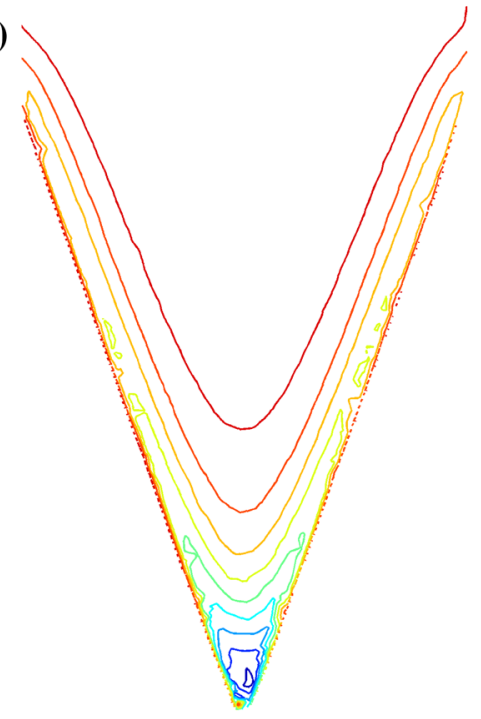




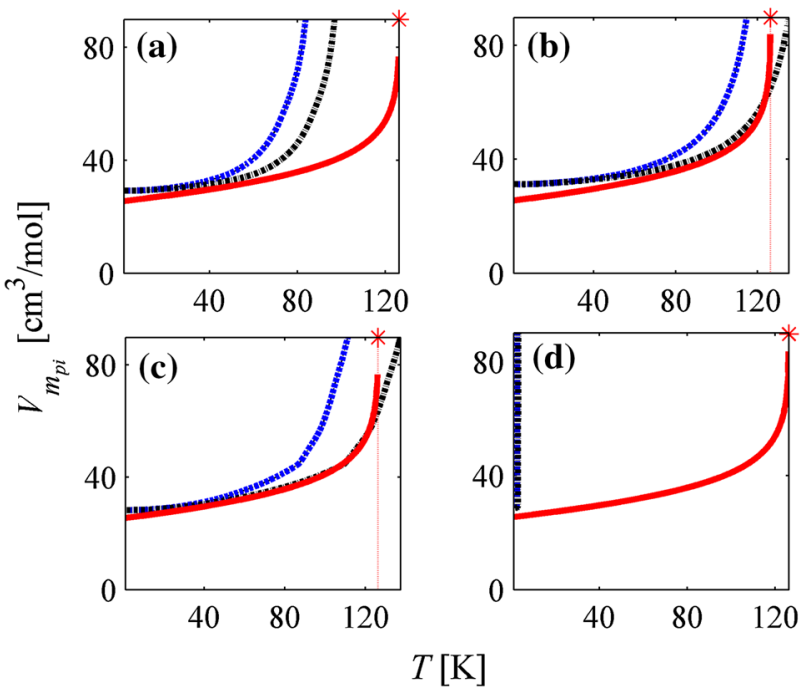

Fig. 8 The calculated temperatures $T_{k}\left(V_{m p i}, p\right)$ (dashed line), $T_{t}$ $\left(V_{m p i}, p\right)$ (dash-dotted line) and liquid nitrogen thermal expansion (solid line) at the volume $V_{m p i}$, for: a $\mathrm{AC}, \mathbf{b} \mathrm{CNC}$, c GS and d SWCNT models. Asterisk corresponds to the critical temperature $T_{c}$ at the critical volume $V_{c}$

On the other hand, for SWCNT model (Fig. 8d) the obtained results give an evidence for a possible mobile mechanism (or other), and so obtained volume immediately exceeds $V_{c}$ and differs from nitrogen liquid expansion curve.

Found with the LBET models and approved in our earlier works (Duda et al. 2013) values for $\zeta$ ratio vary around $0.50-0.48$ and $0.32-0.40$ for the nitrogen adsorption on heterogeneous and homogenous active carbon, respectively. A quite satisfactory agreement of these empirical data and the values calculated in this study may be observed in Fig. 9. The most effective contact ratio was obtained for $\mathrm{CNC}$ and GS materials, but $\zeta$ significantly

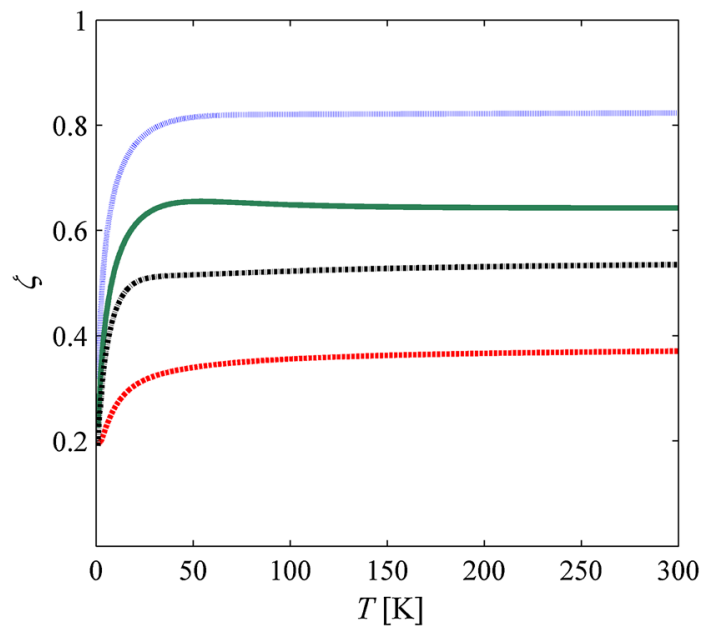

Fig. 9 Calculated intermolecular effective contact ratio $\zeta$ and corresponding temperature $T$, for: $\mathrm{AC}$ (dash-dotted line), $\mathrm{CNC}$ (dotted line), GS (solid line) and SWCNT (dashed line) models exceeds the values obtained in our earlier works. This is due to obvious differences in the structural heterogeneity in both cases. AC structure is geometrically the most consistent and gives the results almost in perfect agreement. Again, as expected, for SWCNT the lowest values of $\zeta$ ratio were obtained. Those results are consistent with determined experimentally weak adsorption on SWCNT (Zhao et al. 2001). Hence, determined values for structure parameter $\zeta$ are in a good agreement with earlier assumed mechanism identification criteria. This consistency of the $\zeta$ values, evaluated with the newly derived formulas and obtained on the basis of experimental examination of adsorption isotherms with LBET models, constitutes $\zeta$ as a validation parameter.

Nevertheless, it has to be pointed out that below a freezing temperature this description is inadequate, since the energy in Eq. (26) is calculated with $P V T$ relationship, dedicated for liquid bulk phase only. However, the attention should be also paid to the invariance of $\zeta$ parameter, that may be observed over a certain values of $T<T_{B}$, see Table $2, \zeta_{\text {const }}$. Let us remind that $\zeta$ is really important parameter that provides information on the pore shape and gets an intuitive view on the material structure. Thus, the way the particle detects structure diversity (pore shape structure $\zeta$ ) became an invariant to the temperature parameter, for the specific pair adsorbate-adsorbent. Therefore, it may make it possible to evaluate the properties of another system, without any additional experimental measurements. This may be achieved by interrelation of a two particles (i.e. by volume, diameter) and then application in Eq. (26). Moreover, it may be also exploited, to improve identification of the adsorbent structure, by simultaneous fitting of two or more isotherms measured at

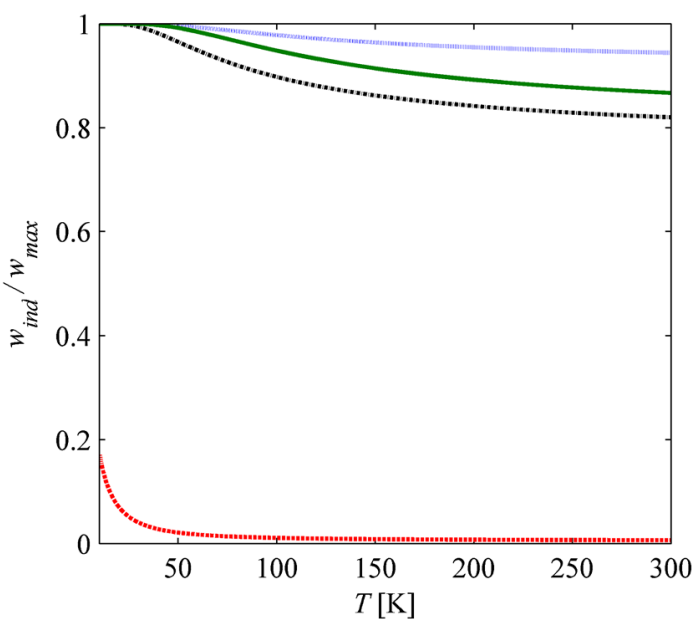

Fig. 10 Ratio $w_{\text {ind }} / w_{\max }$, implying predomination of the particular mechanism, at the temperature $T$ range $0-300 \mathrm{~K}$, AC (dash-dotted line), $\mathrm{CNC}$ (dotted line), GS (solid line) and SWCNT (dashed line) models 
different temperatures for the same pair adsorbateadsorbent.

Calculations were completed with the analysis of adsorption mechanism predomination (Fig. 10), according to Eq. (11) in the temperature range $0-300 \mathrm{~K}$. The most interesting point concerns the normal boiling temperature $T_{B}$, at which nitrogen experimental adsorption is most commonly measured. Hence, at this temperature the localized adsorption mechanism should be assumed for materials where geometrical heterogeneities are close to those modeled in AC, GS and CNC structures. Whereas for SWCNT-like structures other mechanism based mathematical models are certainly more appropriate.

\section{Conclusions}

Presented in this paper novel approach and numerical tools, aimed at qualitative adsorption mechanism identification, are significant development of the works lead in Authors' team. The proposed kinematic description enables for adsorption mechanisms identification, in comparison to the adsorbate critical properties. It was shown that the real particles temperature distribution comes from the thermodynamic equilibrium, corresponding to kinematically determined adsorbate particles volume distribution.

The obtained results are sufficient to get an insight into the adsorption mechanism, and hence performed calculations provide a reliable picture of individual particles movement near the solid surface. In particular, it was shown, that substantial geometrical heterogeneity generates localized attractive adsorption sites, whereas small surface deformations results in mainly possible mobile adsorption.

The numerical tool is also comprehensive in terms of model complexity, since it has been shown that geometrical constraints mainly influence calculations efficiency. Therefore, the properties may be evaluated also even if more than one localized adsorption site is expected.

It has been also demonstrated, that nitrogen volume in the adsorption phase up to the critical volume, may be properly approximated as liquid phase volume. However, the shape of the particle movement significantly differs from a sphere-like space. In order to check the description consistency, the texture parameter $\zeta$ was constituted as validation parameter. It was shown, that the comparison of the results derived from new formulas and on the basis of experimental examination of adsorption isotherms with LBET models, provides the approach validation. It should be also stressed that $\zeta$ becomes temperature invariant over the boiling temperature and it may bring benefits in further researches according to the discussion presented in the paper.
Analysis of the mechanisms predomination with $w_{\text {ind }}$ l $w_{\text {max }}$ ratio is of practical importance. It enables to select the appropriate mathematical model not only intuitively, but on the basis of the calculations performed for the modeled geometrical heterogeneities (e.g. similar as in AC, GS and CNC structures). In particular the results shown in our study suggest that nitrogen adsorption up to boiling temperature is of localized nature in the tested microporous structures of different shape, except for nanotubes.

Acknowledgments The research is led within the AGH University of Science and Technology Grant No. 11.11.210.213.

\section{Compliance with ethical standards}

Conflict of interest The authors declare no competing financial or non-financial interest.

Open Access This article is distributed under the terms of the Creative Commons Attribution 4.0 International License (http://creative commons.org/licenses/by/4.0/), which permits unrestricted use, distribution, and reproduction in any medium, provided you give appropriate credit to the original author(s) and the source, provide a link to the Creative Commons license, and indicate if changes were made.

\section{References}

Baowan, D., Thamwattana, N.: Modelling adsorption of a water molecule into various pore structures of silica gel. J. Math. Chem. 49, 2291-2307 (2011)

Berthelot, D.: Sur le mélange des gaz. C. R. Hebd. Seances Acad. Sci. 126, 1703-1855 (1898)

Bird, R.B., Stewart, W.E., Lightfoot, E.N.: Transport Phenomena. Wiley, New York (2001)

CHERIC, C.E. and M.R.C.: Binary Vapor-Liquid Equilibrium Data, http://www.cheric.org/research/kdb (2015)

De Boer, J.H.: Dynamical character of adsorption. Oxford University Press, Oxford (1968)

Dąbrowski, A.: Adsorption-from theory to practice. Adv. Colloid Interface Sci. 93, 135-224 (2001)

Dubinin, M.M.: Fundamentals of the theory of adsorption in micropores of carbon adsorbents: characteristics of their adsorption properties and microporous structures. Pure Appl. Chem. 61, 1841-1843 (1989)

Duda, J.T., Milewska-Duda, J., Kwiatkowski, M., Ziółkowska, M.: A geometrical model of random porous structures to adsorption calculations. Adsorption 19, 545-555 (2013)

Flory, P.J.: Principles of polymer chemistry. Cornell Univesity Press, Ithaca, New York (1953)

Fowler, R.H., Guggenheim, E.A.: Statistical thermodynamics of super-lattices. Proc. R. Soc. London A Math. Phys. Eng. Sci. 174, 189-206 (1940)

Gauden, P.A., Terzyk, A.P., Furmaniak, S.: Models of activated carbons: past, the present and future. Wiadomości Chem. 62, 403-447 (2008)

Hansen, J.P., McDonald, I.R.: Theory of simple liquids, pp. 74-77. Elsevier, Amsterdam (2006)

Hill, T.L.: Statistical mechanics of multimolecular adsorption II. Localized and mobile adsorption and absorption. J. Chem. Phys. 14, 441-453 (1946) 
http://www.jmol.org/: Jmol: an open-source Java viewer for chemical structures in 3D. (2013)

Jagiełło, J., Olivier, J.P.: 2D-NLDFT adsorption models for carbon slit-shaped pores with surface energetical heterogeneity and geometrical corrugation. Carbon 55, 70-80 (2013)

Kang, J.W., Yoo, K.P., Kim, H.Y., Lee, H., Yang, D.R., Lee, C.S.: Development and current status of the Korea Thermophysical Properties Databank (KDB). Int. J. Thermophys. 22, 487-494 (2001)

Kaukonen, M., Gulans, A., Havu, P., Kauppinen, E.: Lennard-Jones parameters for small diameter carbon nanotubes and water for molecular mechanics simulations from van der Waals density functional calculations. J. Comput. Chem. 33, 652-658 (2012)

Kwiatkowski, M., Duda, J.T., Milewska-Duda, J.: Application of the LBET class models with the original fluid state model to an analysis of single, double and triple carbon dioxide, methane and nitrogen adsorption isotherms. Colloids Surf A 457, 449-454 (2014)

Kwiatkowski, M., Wiśniewski, M., Pacholczyk, A.: The application of the fast multivariant fitting procedure of the LBET models to the analysis of carbon foams prepared by various methods from furfuryl alcohol. Colloids Surf. A 385, 72-84 (2011)

Langmuir, I.: The constitution and fundamental properties of solids and liquids. Part I. Solids. J. Am. Chem. Soc. 38, 2221-2295 (1916)

Lastoskie, C.M., Gubbins, K.E.: Characterization of porous materials using DFT and molecular simulation (2000)

Lennard-Jones, J.E.: Cohesion. Proc. Phys. Soc. 43, 461-482 (1931)

Lorentz, H.A.: Ueber die Anwendung des Satzes vom Virial in der kinetischen Theorie der Gase. Ann. Phys. 248, 127-136 (1881)

Milewska-Duda, J., Duda, J.T.: Handling of a non-BET adsorption with the LBET model. Langmuir 17, 4548-4555 (2001)
Milewska-Duda, J., Duda, J.T.: High-accuracy PVT Relationships for Compressed Fluids and their Application to BET-like Modelling of $\mathrm{CO} 2$ and $\mathrm{CH} 4$ Adsorption. Adsorpt. Sci. Technol. 25, 543-559 (2009)

Milewska-Duda, J., Duda, J.T., Jodłowski, G.S., Kwiatkowski, M.: Model for multilayer adsorption of small molecules in microporous materials. Langmuir 16, 7294-7303 (2000)

Reid, R.C., Prausnitz, J.M., Poling, B.E.: The properties of gases and liquids. McGraw-Hill, New York (1987)

Robinson, M., Marks, N.A.: NanoCap: A framework for generating capped carbon nanotubes and fullerenes. Comput. Phys. Commun. 185, 2519-2526 (2014)

Siderius, D.W., Gelb, L.D.: Extension of the Steele 10-4-3 potential for adsorption calculations in cylindrical, spherical, and other pore geometries. J. Chem. Phys. 135, 084703-1-7 (2011)

Sing, K.S.W., Everett, D.H., Haul, R.A.W., Moscou, L., Pierotti, R.A., Rouquérol, J., Siemieniewska, T.: Reporting physisorption data for gas/solid systems with special reference to the determination of surface area and porosity. Pure Appl. Chem. 57, 603-619 (1985)

Vetere, A.: The Riedel Equation. Ind. Eng. Chem. Res. 30, 2487-2492 (1991)

Volmer, M.A.: Thermodynamische folgerung aus der Zustandsgleichung für adsorbierte Stoffe. Zeitschrift fur Phys. Chemie. 115, 253-261 (1925)

Zhao, J., Buldum, A., Han, J., Lu, J.P.: Gas molecule adsorption in carbon nanotubes and nanotube bundles. Nanotechnology 13, 195-200 (2001) 